\title{
Hyperglycemia, CTCAE
}

National Cancer Institute

\section{Source}

National Cancer Institute. Hyperglycemia, CT CAE. NCI Thesaurus. Code C143552.

A disorder characterized by laboratory test results that indicate an elevation in the concentration of blood sugar. It is usually an indication of diabetes mellitus or glucose intolerance. 